\title{
Preclinical deposition of pathological prion protein PrPSc in muscles of hamsters orally exposed to scrapie
}

\author{
Achim Thomzig, ${ }^{1}$ Walter Schulz-Schaeffer, ${ }^{2}$ Christine Kratzel, ${ }^{1}$ Jessica Mai, ${ }^{1}$ and Michael Beekes ${ }^{1}$
}

${ }^{1}$ Robert Koch-Institut, P26 - Pathogenese und Diagnostik Transmissibler Spongiformer Enzephalopathien, Berlin, Germany. 2Universitätsklinikum Göttingen, Institut für Neuropathologie, Göttingen, Germany.

\begin{abstract}
Recently, pathological prion protein PrPSc, the putative key constituent of infectious agents causing transmissible spongiform encephalopathies (TSEs), was found in muscles of rodents experimentally infected with scrapie and in patients with Creutzfeldt-Jakob disease (CJD). For the assessment of risk scenarios originating from these findings (e.g., alimentary transmission of pathogens associated with bovine spongiform encephalopathy $[\mathrm{BSE}]$ and chronic wasting disease [CWD] via tainted beef and game or iatrogenic dissemination of CJD agent through contaminated surgical instruments) more detailed information about the time course of $\mathrm{PrPSc}^{\mathrm{S}}$ accumulation in muscles at preclinical and clinical stages of incubation is needed. Here we show that $\mathrm{PrP}^{\mathrm{Sc}}$ in muscles of hamsters fed with scrapie can be detected prior to the onset of clinical symptoms, but that the bulk of $\operatorname{PrP}^{S c}$ was deposited late in clinical disease. Additionally, regarding the question of how muscles become invaded, we report on the intramuscular location of $\mathrm{PrPSc}^{\mathrm{Sc}}$ and substantial indications for centrifugal spread of infection from spinal motor neurons to myofibers. Our findings in a well-established animal model for TSEs contribute to a better assessment of the risks for public health emanating from "Prions in skeletal muscle" and provide new insights into the pathophysiological spread of TSE agents through the body.
\end{abstract}

\section{Introduction}

Transmissible spongiform encephalopathies (TSEs) such as scrapie in sheep, bovine spongiform encephalopathy (BSE) in cattle, chronic wasting disease (CWD) in elk and deer, or CreutzfeldtJakob disease (CJD) and its variant form (vCJD) in humans are a group of fatal neurodegenerative disorders of the CNS. Apart from neuropathological features such as gliosis, neuronal cell loss, and spongiform change, these diseases are consistently characterized by the deposition in the CNS of a misfolded form of prion protein $(\mathrm{PrP})$, which is known as $\operatorname{PrPsc}(1)$. According to the prion hypothesis (2), the causative agents of TSEs (so-called "prions") are composed essentially of $\mathrm{PrP}^{\mathrm{Sc}}$, and although the exact molecular structure of these pathogens remains elusive, there is substantial evidence that $\mathrm{Pr}^{\mathrm{Pc}}$ can be used as a reliable biochemical marker for TSE agents (3-6). PrPSc deposition in TSEs is not necessarily confined to the CNS; it has also been observed in a variety of peripheral tissues from donors with experimental and nonexperimental TSEs (1). Irrespective of an incidental observation in experimental caprine scrapie (7), however, muscle tissue from TSE-affected animals or humans was essentially found to be negative for infectivity or $\mathrm{PrPS}^{\mathrm{Sc}}$ in a variety of studies $(6,8-10)$.

The detection of substantial amounts of PrPsc in muscle tissue of mice and hamsters experimentally infected with scrapie (11-13) and of patients with sporadic CJD (14), including those affected by inclusion body myositis (15), has been reported only recently in

Nonstandard abbreviations used: bovine spongiform encephalopathy (BSE); chronic wasting disease (CWD); Creutzfeldt-Jakob disease (CJD); immuno-

histochemistry (IHC); intracerebral lethal doses ( $\left.\mathrm{LD}_{50 \mathrm{c}}\right)$; musculus (M.); paraffinembedded tissue blot (PET blot); prion protein (PrP); transmissible spongiform encephalopathies (TSEs); variant Creutzfeldt-Jakob disease (vCJD).

Conflict of interest: The authors have declared that no conflict of interest exists.

Citation for this article: J. Clin. Invest. 113:1465-1472 (2004).

doi:10.1172/JCI200421083. a series of articles by different research groups. These new findings have revived concerns about the potential risks for public health emanating from possibly unrecognized reservoirs for $\mathrm{PrP}^{\mathrm{Sc}}$ and infectivity in muscles of animals and humans incubating TSEs. Particularly, the theoretical possibility of transmitting pathogens associated with BSE and CWD to humans via tainted beef and game, or that of iatrogenic dissemination of CJD or vCJD agent through contaminated surgical instruments used on muscle tissue, have gained considerable attention. While the assessment of such risk scenarios is certainly difficult, it could greatly benefit from a better knowledge of the time course of $\mathrm{PrP}^{\mathrm{Sc}}$ deposition in muscles at preclinical and clinical stages of incubation.

So far, apart from studies on the tongue of hamsters infected by intralingual injection of TSE agent (13), findings have been reported only on PrPSc or TSE infectivity in muscles from donors already exhibiting clinical symptoms $(7,11,12,14)$. In this article we present data from a time-course study in hamsters fed with $263 \mathrm{~K}$ scrapie, addressing the topical question of preclinical PrPsc deposition in skeletal muscle.

Following up on experimental pathogenesis studies in mice (16-19) and sheep (20), the animal model used in this study has provided key observations on the spread of infection through the body $(4,21-24)$ that have been confirmed in naturally occurring ovine scrapie (25), field cases of BSE (26), orally transmitted BSE in primates (27), and orally transmitted or naturally occurring CWD $(28,29)$. Additionally, more recent reports on the involvement of the sympathetic nervous system in $\mathrm{VCJD}(30)$ and the deposition of PrPSc in muscles of patients with sporadic CJD (14) have shown the relevance of our hamster model for peripheral PrPsc routing in TSE-affected humans.

Here we report that $\mathrm{PrPS}^{\mathrm{Sc}}$ in muscles of hamsters perorally infected with scrapie was already detectable shortly before the onset of clinical symptoms, but that the bulk of PrPSc accumulated only 


\section{Figure 1}

Time course of PrPsc deposition in muscle tissue. Western blot detection of PrP27-30 extracted from different muscles and sciatic nerve of hamsters orally challenged with $263 \mathrm{~K}$ scrapie and sacrificed at the following time points after infection. (A) At 100 days after infection, (B-D) 130 days after infection, (E) onset of clinical signs for scrapie (139-149 days after infection), and (F) at the terminal stage of disease (161-173 days after infection). Lanes with test samples: M1, M. biceps femoris (hindlimb); M2, M. tibialis cranialis (hindlimb); M3, M. triceps brachii (forelimb); M4, M. extensor carpi radialis (forelimb); M5, M. trapezius (shoulder); M6, M. masseter (head); M7, M. psoas major (back); $\mathrm{T}$, tongue; $\mathrm{H}$, heart; $\mathrm{SN}$, sciatic nerve. Lanes with control samples: 1 , proteinase $\mathrm{K}-$ digested brain homogenate from terminally ill scrapie hamsters containing $1 \times 10^{-7} \mathrm{~g}(\mathbf{B}$ and $\mathbf{F})$ or $5 \times 10^{-7} \mathrm{~g}(\mathbf{A}, \mathbf{C}-\mathbf{E})$ brain tissue; 2 , skeletal muscle from an uninfected hamster spiked before extraction with $5 \times 10^{-6} \mathrm{~g}(\mathbf{A}, \mathbf{B}$, and $\mathbf{F})$, $1 \times 10^{-5} \mathrm{~g}(\mathbf{C}$ and $\mathbf{D})$, or $2 \times 10^{-5} \mathrm{~g}$ (E) brain homogenate from terminally ill scrapie hamsters; 3 , skeletal muscle from a mock-challenged hamster sacrificed at 173 days after infection $(\mathbf{F})$. For each stage of incubation representative results are shown. (G) Time scale displaying the mean incubation period and the preclinical and clinical phases of incubation of hamsters orally infected with $263 \mathrm{~K}$ scrapie. Small vertical arrows indicate time points at which animals were screened for PrPsc in muscles. dai, days after infection.
A
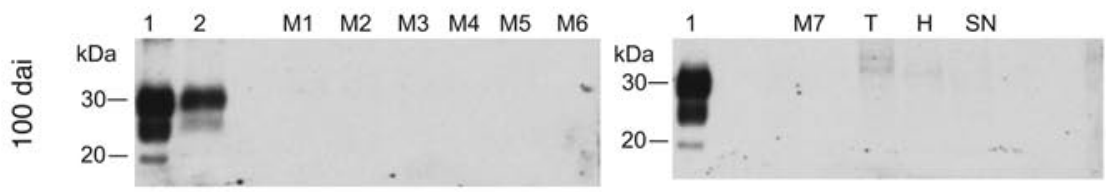

B
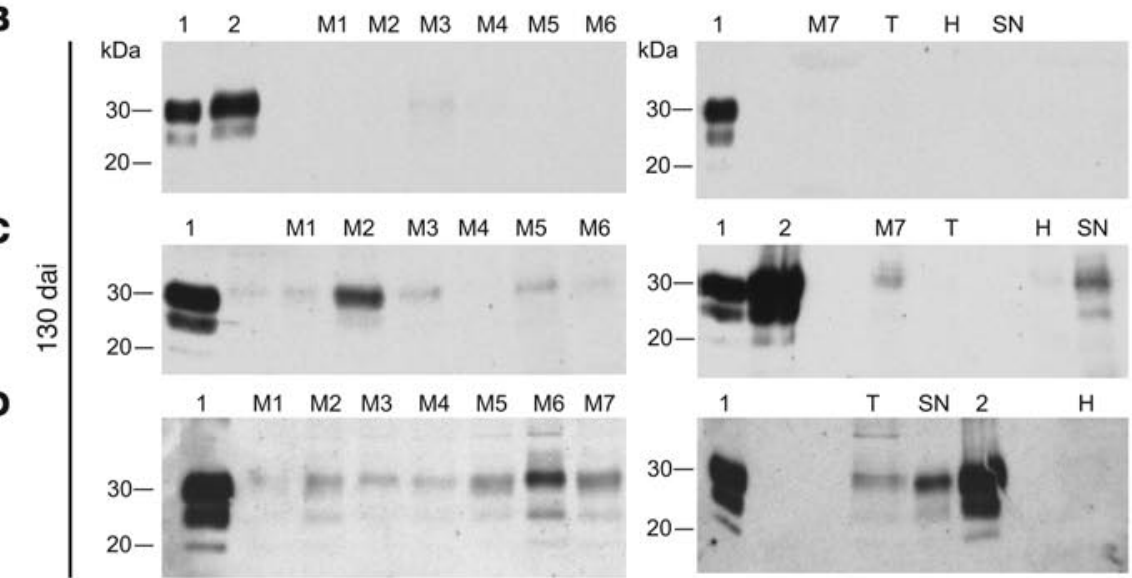

E
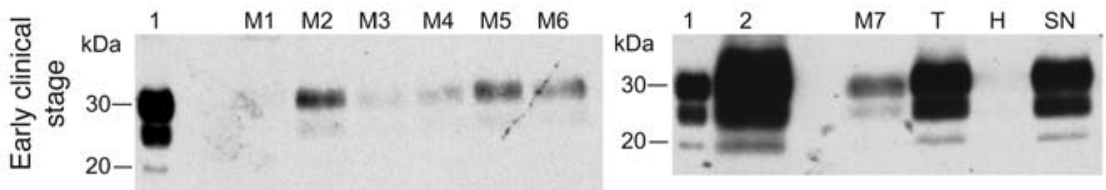

$\mathbf{F}$

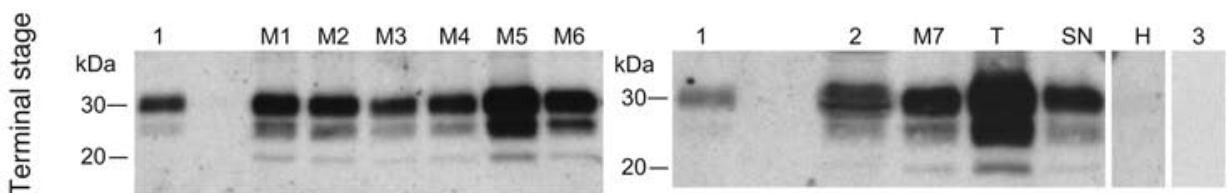

G

Hamsters orally infected with 263K scrapie:

$166 \pm 8$ days until terminal disease
(a) $n=6 ; 100 \mathrm{dai}$
(b) $n=7 ; 130$ dai
(c) $n=5 ; 139,145,145,146,149$ dai
(d) $n=6 ; 161,163,165,170,171,173$ dai

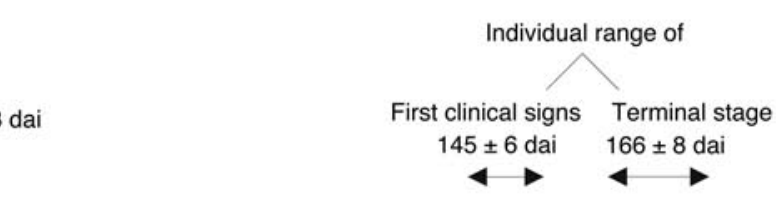

Preclinical phase

Clincal phase

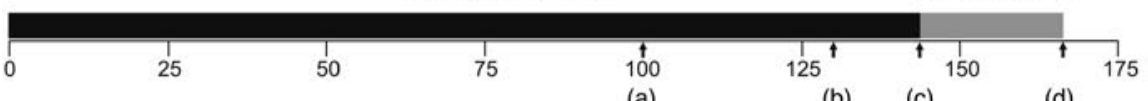

Days after infection (a)

(b)

(c)

(d) late in clinical disease. Improved methods for paraffin-embedded tissue (PET) blotting and immunohistochemistry (IHC) provided detailed information about the intramuscular location of $\mathrm{PrPSc}$ and led to new conceptual insights into the pathophysiology of peripheral $\mathrm{PrPSc}^{\mathrm{Sc}}$ routing.

\section{Results}

Time course of $\mathrm{PrPSc}$ deposition in muscle tissue. To assess whether, and to what extent, the biochemical marker for TSE infectivity, $\mathrm{PrPSc}$, accumulates at preclinical and clinical stages of incubation in skeletal, heart, and tongue muscles of hamsters fed with $263 \mathrm{~K}$ scrapie, the pathological prion protein was visualized by sensitive Western blotting after extraction in the form of PrP27-30 (protease- resistant core of the pathological prion protein $\mathrm{PrPS}^{\mathrm{Sc}}$ ) from tissue samples by a high-yield purification method.

PrP27-30 could not be detected in any of the examined muscle specimens or sciatic nerve samples from six hamsters sacrificed at 100 days after infection (Figure 1A), that is, at about $60 \%$ of the mean incubation period until terminal disease (166 \pm 8 days, expressed as the mean $\pm \mathrm{SD} ; n=20$, with animals also taken from other experimental series). The same holds true for four out of seven animals sacrificed before the onset of scrapie symptoms at 130 days after infection (Figure 1B). In two of the asymptomatic animals culled at 130 days after infection, however, at least one muscle (musculus [M.] tibialis cranialis, Figure 1C, or M. masseter, not shown) was found clearly positive for PrP27-30, and in 


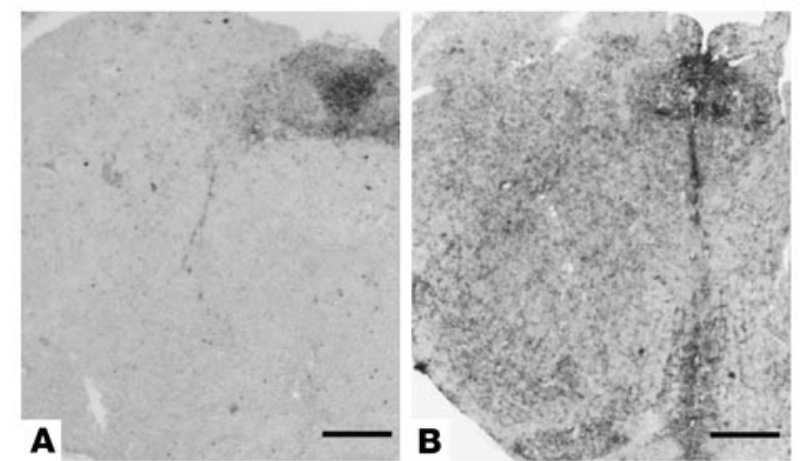

Figure 2

PET blotting of preclinical cerebral PrPsc deposition in the brain stem. The slices comprise the area of the dorsal motor nucleus of the vagus nerve that provides the initial site of PrPSc deposition in the brain of hamsters perorally infected with $263 \mathrm{~K}$ scrapie (A and B). Animals for which all muscles and the sciatic nerve were found negative for PrPsc at 130 days after infection displayed less widespread and intense deposition of $\mathrm{PrPsc}$ in the brain stem (A) than observed in the corresponding brain region (B) of animals already showing PrPsc in their muscle tissue. Scale bars in A and B, $500 \mu \mathrm{m}$.

one other animal of this group PrP27-30 was detected unambiguously in four skeletal muscles (M. tibialis cranialis, M. trapezius, M. masseter, and M. psoas major) as well as in the sciatic nerve (Figure 1D). The signal intensities for PrP27-30 in Figure 1, C and $\mathrm{D}$, suggest that the hamster muscles found to be contaminated at 130 days after infection contained a considerably smaller amount - for most specimens possibly at least fivefold to tenfold - of $\mathrm{PrPS}^{\mathrm{S}}$ than their counterparts taken from terminally ill donors (Figure $1 \mathrm{~F}$ ). The various findings regarding the presence of $\mathrm{PrP}^{\mathrm{Sc}}$ in muscles of different animals at 130 days after infection probably reflect individual variation in the progress of infection. This phenomenon, which leads to different incubation times until terminal disease, is mirrored by the extent of cerebral $\mathrm{PrPSc}^{\mathrm{Sc}}$ accumulation. Animals in which all muscles were found negative for $\mathrm{Pr} P \mathrm{Sc}$ at 130 days after infection (Figure 1B) exhibited less widespread and intense deposition of $\mathrm{PrPSc}^{\mathrm{S}}$ in the brain stem (e.g., at the level of the dorsal motor nucleus of the vagus nerve, which provides the initial site of $\mathrm{PrPS}^{\mathrm{Sc}}$ deposition in the brain of hamsters orally infected with scrapie [ref. 21]; Figure 2A) and other brain regions (not shown) than hamsters already showing $\mathrm{PrP}^{\mathrm{Sc}}$ in their muscle tissue (Figures 1D and 2B).

At the onset of clinical symptoms the observed pattern of PrPSc deposition in muscles became more uniform. Here, the tongue samples (and sciatic nerve specimens) from all examined animals displayed strong signals for PrP27-30 (Figure 1E, lanes T and SN) with an intensity already comparable to that observed in terminal disease (Figure 1F, lanes $\mathrm{T}$ and $\mathrm{SN}$ ). Of the other analyzed muscles from animals at the early clinical stage of scrapie, at least two, and frequently three or four (mostly including the M. tibialis cranialis and the $M$. trapezius), were found to be positive for PrP27-30 (a representative example is given in Figure 1E, lanes M1-M7). These muscle specimens, however, consistently exhibited signal intensities that were still substantially lower than those observed for the corresponding muscles in terminally ill scrapie hamsters (Figure 1F, lanes M1-M7).

At the terminal stage of scrapie all of the examined skeletal muscles and tongue specimens showed a consistent accumulation of
$\mathrm{PrP}^{\mathrm{Sc}}$ as demonstrated by strong PrP27-30 immunostaining in the Western blot. Heart muscle was only very slightly positive in two out of six terminally ill animals (the sample displayed in Figure 1F, lane $\mathrm{H}$, exhibits no signal for PrP27-30). Muscle samples from mock-challenged age-matched control hamsters consistently gave negative results (Figure 1F, lane 3, shows one representative sample: M. extensor carpi radialis). Detection thresholds for $\mathrm{PrP}^{\mathrm{Sc}} /$ PrP27-30 in the Western blot analyses were in the range previously reported (12) as validated in this study by positive controls of muscle tissue from uninfected hamsters spiked with either $5 \times 10^{-6} \mathrm{~g}$, $1 \times 10^{-5} \mathrm{~g}$, or $2 \times 10^{-5} \mathrm{~g}$ of homogenized scrapie-infected hamster brain from terminally ill donors (Figure $1, \mathrm{~A}-\mathrm{F}$, lane 2).

As far as the blotting results shown in Figure 1 allow a comparison of the glycosylation and electrophoretic migration patterns of PrP27-30 from muscles, sciatic nerve, and brain, no significant differences can be discerned despite the divergent nature of these tissues. Our Western blot experiments, however, were primarily designed for the sensitive detection of pathological prion protein. A thorough molecular characterization of muscle-associated $\mathrm{Pr} \mathrm{P}^{\mathrm{Sc}}$, which needs to be performed in future studies, will require the Western blot collation of carefully adjusted amounts of PrP27-30 that produce optimized staining intensities and clearly visible unglycosylated bands.

Intramuscular location of PrPSc. The morphological PET blot analysis of the distribution of proteinase $\mathrm{K}$-resistant $\mathrm{PrP}$ in muscle tissues revealed the deposition of $\mathrm{PrPSc}^{\mathrm{Sc}}$ in muscle fibers (Figure 3, A, D, and E; arrowheads) and nerve fascicles (Figure 3, A and $\mathrm{E}$; arrows). In nerve fascicles, which consistently appeared somewhat less-intensely stained than muscle fibers, the deposition was endoneural (inset in Figure 3E). In muscle fascicles, $\mathrm{PrP}^{\mathrm{Sc}}$ was found in adjacent, as well as topologically distant, muscle fibers (prominent in Figure 3D; arrowheads), similar to a distribution that would be expected for motor units innervated by individual motor neurons in the spinal cord $(31,32)$. Within individual muscle fibers, $\mathrm{PrP}^{\mathrm{Sc}}$ was found predominantly subsarcolemmal in the region of the fiber surface but also granularly scattered (Figure 3D). In some fibers $\mathrm{PrP}^{\mathrm{Sc}}$ seems to be associated with structures reminiscent of neuromuscular junctions as exemplarily indicated by the strong staining at the right border of the myofiber marked by an asterisk (Figure $3 \mathrm{~A}$ and inset).

To corroborate these PET blot findings, we tried to visualize $\mathrm{PrP}^{\mathrm{Sc}}$ deposition in muscles by IHC. To reliably avoid misleading IHC immunostaining in muscle samples, a specifically designed protocol needed to be established that included pretreatment of specimens by hyaluronidase and proteinase $\mathrm{K}$. The immunohistochemical examinations confirmed the subsarcolemmal location of $\mathrm{PrP}^{\mathrm{Sc}}$ as well as the protein's granularly scattered occurrence within muscle fibers (Figure $3 \mathrm{~F}$ and inset). Because of the lower sensitivity of IHC as compared with PET blotting and other technical difficulties, however, immunohistochemical detection of PrPSc could be achieved only in a small proportion of the analyzed samples (Figure 3F). In the majority of specimens found to be positive by PET blotting, IHC produced negative results (Figure 3C). Muscle samples from mock-challenged control hamsters consistently gave negative PET blot and IHC results (not shown).

Infectivity in muscle tissue. To formally verify that $\mathrm{PrP}^{\mathrm{Sc}}$ found in the muscles of our model animals correlates with TSE infectivity, we performed a supplementary series of bioassays in which hamsters were intracerebrally challenged with homogenized muscle tissue from terminally ill scrapie hamsters. Four different skeletal 


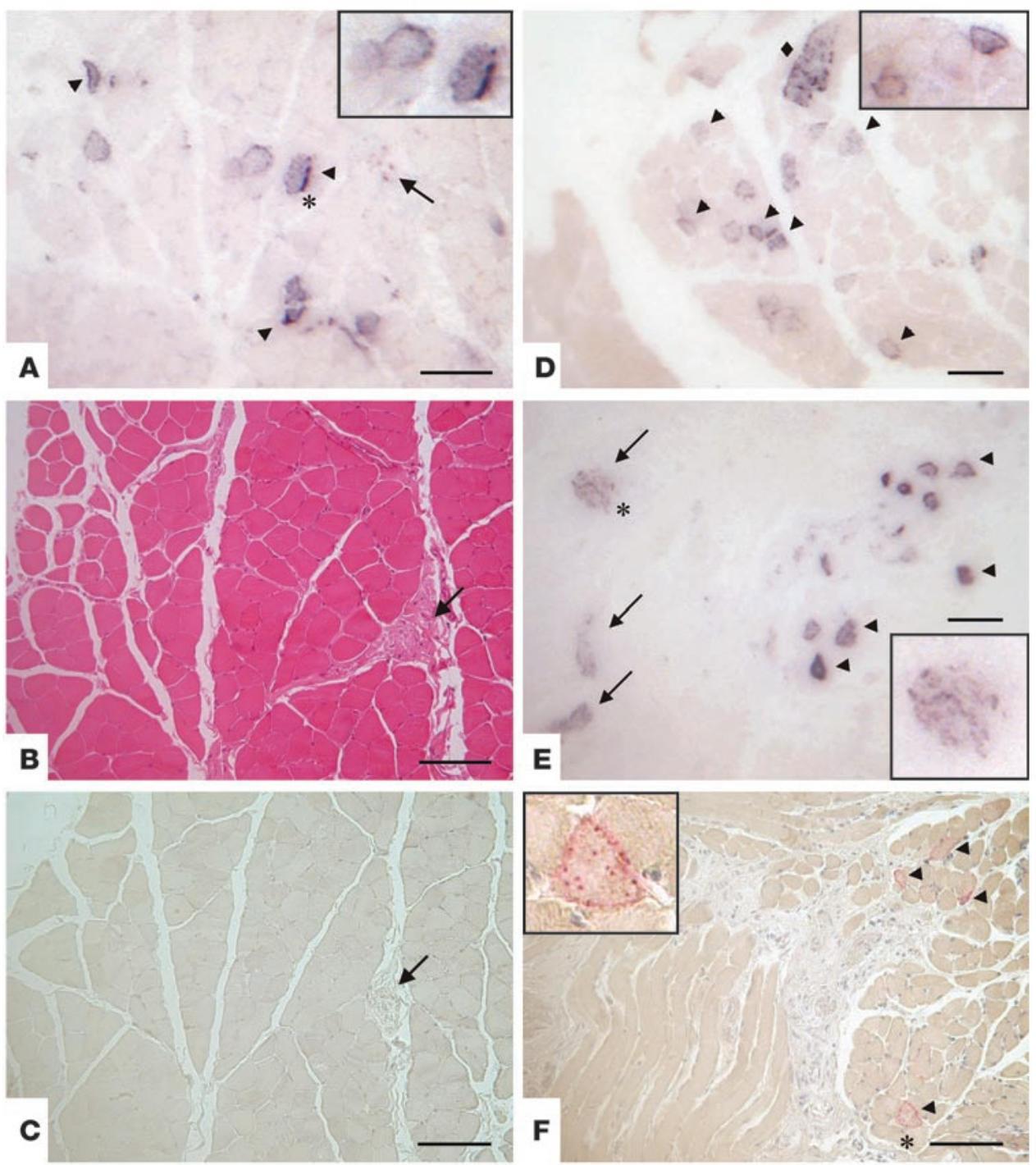

Figure 3

Intramuscular location of PrPSc in terminally ill $263 \mathrm{~K}$ scrapie hamsters visualized by PET blotting and IHC. (A) PET blot from M. triceps brachii. Individual muscle fibers display deposition of PrPsc predominantly subsarcolemmal in the region of the fiber surface, but PrPSc is also scattered within fibers (arrowheads). At some myofibers PrPSc immunostaining exhibits a structural pattern reminiscent of neuromuscular junctions (asterisk and inset). Only minor PrPsc deposits are visible within nerve fascicles (arrow). (B) Adjacent section to $\mathbf{A}$, stained with H\&E; arrow: nerve fascicle. (C) Adjacent section to A, immunohistochemically stained with mAb 3F4 without detectable PrPSc deposition; arrow: nerve fascicle. (D) PET blot from M. masseter; PrPSc in adjacent as well as topologically distant muscle fibers (arrowheads), distributed in a pattern reminiscent of that expected for motor units innervated by individual neurons in the spinal cord. Note: Tangentially cut muscle fiber (rhomb) with strong granularly scattered immunolabeling. Reproduction in higher magnification (inset: photomicrograph from a different section) illustrates prominent subsarcolemmal localization of PrPsc deposition in myofibers. (E) PET blot from lingual muscle; distinct endoneural PrPsc accumulation in nerve fascicles (arrows). One fascicle (asterisk) is reproduced in higher magnification in the inset. The intensity of PrPSc deposition in muscle fibers of the tongue (arrowheads) seems to be stronger than that observed in other muscles. (F) Sample from lingual muscle immunohistochemically stained with mAb 3F4; granular immunostaining illustrates subsarcolemmal as well as scattered PrPSc deposition in muscle fibers (arrowheads; inset: higher magnification of fiber marked by an asterisk). Scale bars in A-F, $100 \mu \mathrm{m}$.

scrapie as verified by Western blot detection of $\mathrm{PrPSc} / \mathrm{PrP} 27-30$ in their brains (Figure 4, lane 1).

The results of the infectivity bioassay are summarized in Table 1. Mortalities and incubation times for the recipients in the bioassay demonstrated a consistent presence of infectivity in the range of about $2 \times 10^{3}$ to $10 \times 10^{3}$ $50 \%$ intracerebral lethal doses $\left(\mathrm{LD}_{50 \mathrm{ic}}\right)$ per gram of tissue, except for the M. triceps brachii from the forelimb, which exhibited a surprisingly low infectivity titer of only $100-300 \mathrm{LD}_{50 \mathrm{ic}} / \mathrm{g}$. Three hamsters inoculated with tissue from this muscle failed to exhibit clinical symptoms until 350 days after infection. Consistent with the low amount of infectivity revealed by bioassay for the inocula of M. triceps brachii, however, the inconspicuous reporter animals were subclinically infected as shown in Figure 4, lane 2.

The lingual muscle showed the highest infectivity titer of the examined specimens, a finding consistently mirrored by the Western blot results. The determined infectivity titers appear somewhat below the level expected on the basis of the observed PrPSc immunostaining intensities, however. The detected amount of PrP27-30 in the muscles from terminally ill hamsters is comparable to, or even higher than, that present in $5 \times 10^{-6} \mathrm{~g}$ of homogenized scrapie-infected hamster brain (Figure 1F, lanes M1-M7 and $\mathrm{T}$ versus lane 2 ). As previously discussed elsewhere (12), this would correspond to an infectivity titer of approximately $10^{5} \mathrm{LD}_{50 \mathrm{ic}} / \mathrm{g}$ if $\mathrm{PrP}^{\mathrm{Sc}}$ in muscles correlated with infectivity at a ratio similar to that observed in the brain or spinal cord of $263 \mathrm{~K}$-infected hamsters. Thus, the bioassay findings possibly provide an underestimation of the infectivity in muscles since the titrated inocula, after digestion of samples in collagenase A, had been heated for technical reasons (see Methods) to $80^{\circ} \mathrm{C}$ before intracer-

muscles (M. biceps femoris, M. triceps brachii, M. trapezius, M. psoas major) and the lingual muscle from two donor animals were titrated for infectivity. All reporter animals of which the incubation times were used to calculate infectivity titers succumbed to ebral administration. Theoretically, it appears conceivable that this treatment, which somewhat differs from conventional ways of tissue homogenization (11), may have partially inactivated the infectious scrapie agent originally present in situ. 


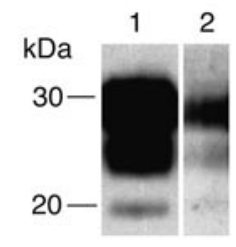

Figure 4

Testing of bioassay reporter animals for cerebral PrPSc deposition. Western blot detection of PrP27-30, the protease-resistant core of PrPsc, in proteinase $\mathrm{K}$-digested brain homogenates from hamsters intracerebrally inoculated with muscle samples from terminally ill donors orally challenged with $263 \mathrm{~K}$ scrapie. Lane 1: representative finding for reporter animals that succumbed to scrapie; sample from a terminally ill hamster challenged with homogenized M. biceps femoris (hindlimb) containing $10^{-6} \mathrm{~g}$ brain tissue. Lane 2: representative finding for reporter animals that failed to show scrapie symptoms until 350 days after infection; sample from a subclinically infected hamster challenged with homogenized M. triceps brachii (forelimb) containing $10^{-3} \mathrm{~g}$ brain tissue.

Comprehensive inactivation studies, however, failed to reveal a decrease of infectivity in scrapie brain homogenate from $263 \mathrm{~K}$ infected hamsters heated under the conditions used in our titration experiments, that is, for 10 minutes to a temperature of $80^{\circ} \mathrm{C}(33)$. In light of the pertinent report by Rohwer (33), the sensitivity of the bioassay would appear unlikely to have been reduced - if at all - by more than 0.5 logs due to our method for the preparation of inocula. Therefore, the unexpectedly low infectivity titers found in muscle samples from our model animals may point to higher $\mathrm{PrPs} /$ infectivity ratios in the examined specimens than previously established for CNS tissue (3-4). This explanation would also be consistent with the astonishingly low levels of infectivity measured in the $\mathrm{M}$. triceps brachii despite the substantial amounts of PrP27-30 detected in that muscle.

\section{Discussion}

Our studies demonstrate that $\mathrm{PrP}^{\mathrm{Sc}}$ can be detected in skeletal muscles of hamsters fed with scrapie before the animals show clinical signs of disease. Preclinical detection of pathological prion protein in muscle tissues was confined to relatively late stages of the asymptomatic phase of incubation, however. The bulk of muscle-associated $\mathrm{PrP}^{\mathrm{Sc}}$ accumulated during clinical disease and all examined skeletal muscles and tongue specimens from terminally ill hamsters contained substantial amounts of $\mathrm{PrPSc}^{\mathrm{Sc}}$ in accordance with previous observations (12). The accumulation of $\mathrm{PrPSc}$ found in the latter group of animals was accompanied by the consistent presence of at least $2 \times 10^{3}$ to $10 \times 10^{3} \mathrm{LD}_{50 \mathrm{ic}}$ of scrapie agent per gram of tissue in the $\mathrm{M}$. biceps femoris, M. trapezius, M. psoas major, and lingual muscle. PET blot and IHC examinations corroborated the Western blot findings in our hamsters and provided new detailed information on the distribution and location of $\mathrm{PrPSc}$ in muscle tissue. Since the animal model used in this study in the past has been frequently found to exhibit key pathogenetic features of peripheral $\mathrm{PrPSc}^{\mathrm{Sc}}$ routing $(4,12,21-24)$ in several different alimentarily acquired and naturally occurring TSEs of animals and humans (14, 25-30), the findings reported here may well have several relevant implications:

Pathophysiology of peripheral PrPSc deposition in muscles. In experiments with transgenic mice exhibiting a four- to eightfold overexpression of the cellular isoform of the prion protein $\left(\mathrm{PrP}^{\mathrm{C}}\right)$ in myofibers under the control of myocyte-specific promoters, Bosque et al. (11) showed intrinsic replication of infectivity in muscles injected with scrapie agent. Here, with the more natural route of peroral infection, we have provided direct experimental evidence for the deposition of $\mathrm{PrP}^{\mathrm{Sc}}$ in myofibers, particularly subsarcolemmally, and found that the deposition of this protein in muscles is also endoneurally associated with nerve fibers. Furthermore, the distribution patterns of $\mathrm{PrP}^{\mathrm{Sc}}$ observed in muscles conspicuously resembled those known for the innervation of motor units $(31,32)$. Taken together, these findings are highly indicative of spread of infection from motor neurons in the spinal cord via axonal projections to neuromuscular junctions and on from there postsynaptically into muscle fibers. The results from the Western blot studies clearly show that $\mathrm{PrPsc}^{\mathrm{sc}} \mathrm{can}$ be detected in muscles only shortly before the onset of clinical symptoms, that is, at a time point when the CNS is already heavily loaded with $\mathrm{PrP}^{\mathrm{Sc}}$ and infectivity. This, again, would most plausibly be explained by projections of the peripheral nervous system mediating centrifugal infection of muscles from the spinal cord. Further time-course studies will be necessary, however, to validate the proposed neural spreading pathway and to elucidate, or rule out, the relevance of alternative mediators for muscle invasion such as lymph and blood or neural pathways linked with the centripetal ascension of TSE agent to the CNS.

\section{Table 1}

Detection of infectivity in muscles of terminally ill hamsters orally infected with $263 \mathrm{~K}$ scrapie

\begin{tabular}{|c|c|c|c|c|}
\hline Donor & Sample & $\begin{array}{l}\text { Mortality due to scrapie } \\
\text { (until } 350 \text { dai) }\end{array}$ & Incubation time (days) & Infectivity titer $\left(10^{3} \mathrm{LD}_{50 i \mathrm{ic}} / \mathrm{g}\right)$ \\
\hline 1 & Hindlimb (M. biceps femoris) & $4 / 4$ & $131,166,215,215$ & 2.0 \\
\hline 2 & Hindlimb (M. biceps femoris) & $5 / 5$ & $134,136,180,343,349$ & 2.0 \\
\hline 1 & Forelimb (M. triceps brachii) & $5 / 5$ & $187,225,254,260,329$ & 0.3 \\
\hline 2 & Forelimb (M. triceps brachii) & $2 / 5$ & 267,338 & 0.1 \\
\hline 1 & Shoulder (M. trapezius) & $5 / 5$ & $113,134,208,232,236$ & 10.9 \\
\hline 2 & Shoulder (M. trapezius) & $5 / 5$ & $124,127,131,166,243$ & 5.7 \\
\hline 1 & Back (M. psoas major) & $5 / 5$ & $120,145,208,211,232$ & 7.6 \\
\hline 2 & Back (M. psoas major) & $5 / 5$ & $131,131,134,141,280$ & 2.6 \\
\hline 1 & Lingual muscle & $2 / 2$ & 120,131 & 8.1 \\
\hline 2 & Lingual muscle & $3 / 3$ & $121,139,164$ & 11.7 \\
\hline
\end{tabular}

Aliquots $(50 \mu \mathrm{l})$ of homogenized muscle samples from terminally ill hamsters orally challenged with $263 \mathrm{~K}$ scrapie were inoculated intracerebrally into groups of two to five recipients. Mortality is shown until 349 days after infection, and the experiment was terminated at 350 days after infection. 
Risks for public health and protection of consumers and patients. What conclusions regarding the risks for public health emanating from "Prions in skeletal muscle" can be drawn from the findings described in this report?

For the reasons outlined above, the experimental paradigm used in our study is likely to provide a relevant model on the issue of muscle contamination. It probably reflects a worst-case rather than a best-case scenario, however, because the levels of infectivity and $\mathrm{PrPSc}$ produced in the CNS and other neural tissues of $263 \mathrm{~K}$ scrapie-infected hamsters generally tend to be higher than in nonexperimental TSEs. Indeed, other than for caprine scrapie (7) or sporadic CJD (14), no infectivity or $\mathrm{PrP}^{\mathrm{Sc}}$ has been detected so far in skeletal muscles of cattle with BSE (10).

If the findings in the hamster model were confirmed, to a varying degree, in ovine scrapie, BSE, or CWD, significant deposition of $\mathrm{PrPS}^{\mathrm{Sc}}$ in muscles of affected sheep, cattle, and elk or deer would be expected to occur only at relatively late stages of preclinical infection, with the bulk of muscle-associated $\mathrm{PrP}^{\mathrm{Sc}}$ accumulating during clinical disease. At those stages of incubation, however, the TSE routine tests currently in use should allow both an identification of affected animals (34) with high reliability and their safe disposal. Thus, our findings strongly support the schemes for active TSE testing of animals that have already been implemented in several countries as an efficient precautionary measure to prevent muscle tissue potentially contaminated with infectious TSE agent from entering the food chain.

In addition to recent findings in patients with sporadic CJD (14), our observations in the hamster model corroborate concerns that muscles from inconspicuous patients preclinically incubating CJD or vCJD may represent a potential risk tissue for the nosocomial transmission of human TSE agents. Epidemiological evidence substantiating this hypothetical hazard has not been reported, however, and in any case the risk can be considered as effectively addressed by comprehensive precautionary measures already implemented for infection control in nosocomial settings. Particularly, recommended procedures for the routine maintenance (cleaning, disinfection, and sterilization) of surgical instruments $(35,36)$ aimed at the reliable removal and inactivation of unrecognized contaminations with infectious agents are expected to substantially reduce iatrogenic risks possibly originating from TSE agents in muscles - if they are firmly observed.

Since 2002, a considerable body of new data on $\mathrm{PrP}^{\mathrm{Sc}}$ and TSE infectivity in muscles of experimentally infected rodents and patients with sporadic CJD has been compiled (11-14). It remains to be examined in future studies whether, and to what extent, these findings can be transferred to nonexperimental scrapie, BSE, and CWD, or to vCJD in humans. Special attention should be paid to food products containing ruminant or cervid tongue, given the observations reported here and elsewhere (13). In any case, the data available so far leave no doubt that, 40 years after Pattison and Millson (7) reported finding the scrapie agent in muscle tissue of an experimentally infected goat, a topical field of TSE research has been (re)opened.

\section{Methods}

\section{Animal inoculations and preparation of tissue samples}

All animal experiments were performed in accordance with European and German legal and ethical regulations and approved by the responsible review boards and authorities. Outbred Syrian hamsters were perorally infected or mock-challenged with a single dose of homogenized $263 \mathrm{~K}$ scrapie or normal hamster brain (corresponding to $10 \mathrm{mg}$ of tissue), respectively, as previously described (4). Scrapie-infected animals were humanely sacrificed for Western blot examinations at 100 days after infection $(n=6)$, at 130 days after infection $(n=7)$, at the onset of at least two clinical signs for scrapie, such as tremor of head or whole body, incoordination of gait, or difficulty in rising up from a supine position $(n=5: 139,145,145,146$, and 149 days after infection), and at the terminal stage of disease ( $n=6: 161,163$, $165,170,171$, and 173 days after infection). For PET blotting of muscle samples and infectivity bioassays, five additional hamsters in the terminal stage of scrapie were sacrificed at 171, 173 and 174 and at 163 and 168 days after infection, respectively. Control animals (mock-challenged age-matched hamsters) were sacrificed at corresponding time points. Heart muscle (apex), lingual muscle (approximately $5 \mathrm{~mm}$ from the tip of the tongue), sciatic nerves, and seven different skeletal muscles from the hindlimb (M. biceps femoris; M. tibialis cranialis), the forelimb (M. triceps brachii [caput laterale]; M. extensor carpi radialis), the shoulder (M. trapezius), the head (M. masseter), and the back (M. psoas major) were dissected for Western blot examinations, and visible nerve fibers were removed from the muscle specimens. All samples for Western blot analyses were cut into small pieces and stored at $-80^{\circ} \mathrm{C}$ until examination. The mass of the muscle samples ranged from approximately 40 to approximately $100 \mathrm{mg}$, and that of the sciatic nerves from approximately 5 to approximately $10 \mathrm{mg}$. Sampling from hamsters sacrificed for PET blot analysis and infectivity bioassays was performed as described below. To avoid cross contamination, instruments used for the preparation of samples were carefully cleaned after removal and processing of each specimen.

\section{Western blot examinations}

Muscle and nerve specimens to be examined by Western blotting were processed as previously described (12) for the extraction of $\mathrm{PrP}^{\mathrm{Sc}}$ in the form of $\operatorname{PrP} 27-30(3,37)$; the final pellets were resuspended in $15 \mu \mathrm{l}$ of $2 \times$ sample loading buffer and heated to $100^{\circ} \mathrm{C}$ for $5 \mathrm{~min}$ utes before Western blotting using the anti-PrP mAb 3F4 (38).

Proteinase K-digested homogenate from scrapie hamster brains, used as a PrP27-30 reference in the Western blotting analyses, was prepared as outlined previously $(12,37)$.

SDS-PAGE and Western blot analyses were performed as described elsewhere $(3,37)$ with recently published modifications (12). PrP signals were visualized on a X-OMAT AR (Kodak; Sigma-Aldrich, Steinheim, Germany) film. Film was exposed for 30 minutes.

\section{PET blot examinations}

PET blotting of brain samples. Brains were immediately removed from sacrificed hamsters and immersed for 48 hours in $4 \%$ phosphate-buffered formalin as described by Schulz-Schaeffer et al. (39). After treatment with formic acid for 1 hour at room temperature, brains were placed in $4 \%$ phosphate-buffered formalin for a further 24 hours and subsequently trimmed coronally into 2 - to 3-mm-thick slices. Samples were embedded in paraffin wax, and PET blotting was carried out as described (39), with slight modifications. Six-micrometer microtome slices were mounted onto nitrocellulose membranes and dried flat at $50^{\circ} \mathrm{C}$ for 16 hours. After washing, sections were digested with $250 \mu \mathrm{g} / \mathrm{ml}$ proteinase 
$\mathrm{K}$ (Roche Molecular Biochemicals, Mannheim, Germany) in PK digestion buffer containing $10 \mathrm{mM}$ Tris- $\mathrm{HCl}$ (pH 7.8), $100 \mathrm{mM}$ $\mathrm{NaCl}$, and $0.1 \%$ Brij 35 for 8 hours at $55^{\circ} \mathrm{C}$. Sections were then denatured in $3 \mathrm{M}$ guanidine thiocyanate and blocked with $0.2 \%$ casein before labeling with mAb 3F4.

PET blotting of muscle samples. Morphological analysis of the distribution and location of $\mathrm{PrP}^{\mathrm{Sc}}$ in muscle tissue by PET blotting was performed on lingual muscle (approximately $5 \mathrm{~mm}$ from the tip of the tongue) and the seven different skeletal muscles specified above. The specimens were taken from three hamsters that developed terminal symptoms of scrapie after peroral infection at 171,173 , and 174 days after infection and from corresponding mock-infected controls. Animals were transcardially perfused with periodate-lysine-paraformaldehyde (22), and removed tissue samples were immersed additionally for 5 hours in periodatelysine-paraformaldehyde. After incubation for 8 hours at $4^{\circ} \mathrm{C}$ in phosphate-buffered saline, muscle samples were transferred to $70 \%$ ethanol, processed in a tissue processor, and subsequently embedded in paraffin wax. For PET blot examinations of muscle tissue (2- to 3 - $\mu$ m-thick sections), modifications of the original protocol (39) were necessary to remove connective tissue. After prewetting blots with TBST (10 mM Tris-HCl, pH 7.8, $100 \mathrm{mM}$ $\mathrm{NaCl}, 0.05 \%$ Tween 20 ) sections were digested with $1.5 \mathrm{mg} / \mathrm{ml}$ collagenase A (Roche Molecular Biochemicals) in a buffer containing $10 \mathrm{mM}$ Tris- $\mathrm{HCl}, \mathrm{pH}$ 7.8, $100 \mathrm{mM} \mathrm{NaCl}, 100 \mathrm{mM} \mathrm{CaCl}_{2}$, and $0.1 \%$ Brij 35 for 30 minutes at $60^{\circ} \mathrm{C}$ followed by digestion using $250 \mu \mathrm{g} / \mathrm{ml}$ proteinase $\mathrm{K}$ (Roche Molecular Biochemicals) in PK digestion buffer (see above) for 8 hours at $55^{\circ} \mathrm{C}$.

\section{Immunohistochemical examinations}

Examination of the distribution and location of $\mathrm{PrP}^{\mathrm{Sc}}$ in muscle tissue by IHC was performed on 2- to 3 - $\mu \mathrm{m}$-thick sections of paraffin-embedded muscle specimens prepared as described above (see PET blot examinations). Sections were pretreated with $2 \mathrm{mg} / \mathrm{ml}$ hyaluronidase (Sigma-Aldrich) in sodium acetate buffer ( $\mathrm{pH} 5.2 ; 21 \mathrm{mM}$ acetic acid, $79 \mathrm{mM}$ sodium acetate) for 30 minutes at $37^{\circ} \mathrm{C}$ and then exposed at the same temperature and for the same time to $50 \mu \mathrm{g} / \mathrm{ml}$ proteinase $\mathrm{K}$ in TBS $(10 \mathrm{mM}$ Tris $\mathrm{HCl}, 133 \mathrm{mM} \mathrm{NaCl}, \mathrm{pH}$ 7.4) containing $2 \mathrm{mM} \mathrm{CaCl}_{2}$. After incubation with the mAb 3F4 (1:500) and secondary alkaline phosphatase-coupled goat anti-mouse Ab (1:500; Dako, Hamburg, Germany) for 90 minutes each, immunostaining was performed using new fuchsine as chromogen. Subsequently, slides were counterstained with hemalaun.

1. Prusiner, S.B. 1998. Prions. Proc. Natl. Acad. Sci. U. S. A. 95:13363-13383.

2. Prusiner, S.B. 1982 . Novel proteinaceous infectious particles cause scrapie. Science. 216:136-144.

3. Beekes, M., Baldauf, E., and Diringer, H. 1996. Sequential appearance and accumulation of pathognomonic markers in the central nervous system of hamsters orally infected with scrapie. J. Gen. Virol. 77:1925-1934.

4. Baldauf, E., Beekes, M., and Diringer, H. 1997. Evidence for an alternative direct route of access for the scrapie agent to the brain bypassing the spinal cord. J. Gen. Virol. 78:1187-1197.

5. Ironside, J.W., Head, M.W., Bell, J.E., McCardle, L., and Will, R.G. 2000. Laboratory diagnosis of variant Creutzfeldt-Jakob disease. Histopathology. 37:1-9.

6. Wadsworth, J.D., et al. 2001. Tissue distribution of protease resistant prion protein in variant Creutzfeldt-Jakob disease using a highly sensitive immunoblotting assay. Lancet. 358:171-180.

\section{Infectivity bioassay}

Infectivity titers in selected muscle specimens were assessed by an incubation time-interval assay $(40,41)$. For this purpose, four different skeletal muscles (M. biceps femoris, M. triceps brachii, M. trapezius, and M. psoas major) and the tip of the tongue were removed from two terminally ill scrapie hamsters sacrificed at 163 and 168 days after infection. Tissue specimens were homogenized in a way similar to that for the Western blot examinations. After removal of visible nerve fibers, samples were washed three times in TBS and incubated in a rocking device at $37^{\circ} \mathrm{C}$ for 3.5 hours in TBS containing $2 \mathrm{mM} \mathrm{CaCl}_{2}$ and $0.25 \%$ collagenase A (Roche Molecular Biochemicals). Collagenase A digestion was performed in a total volume of $500 \mu \mathrm{l}$ per sample. Following ultrasonification, samples were heated to $80^{\circ} \mathrm{C}$ for 10 minutes in order to accomplish inactivation of the enzyme and of accidental microbial contaminations prior to intracerebral inoculation. Aliquots $(50 \mu \mathrm{l})$ of the muscle homogenates were intracerebrally inoculated into groups of two to five recipient hamsters each, and the amount of infectivity in the samples was assayed as previously described (3-4) using the incubation times shown in Table 1 (mean error of assay \pm 0.4 logs). Proteinase K-digested homogenates were prepared from the brains of all reporter animals (including those that failed to show scrapie symptoms), as described elsewhere $(12,37)$, and subjected to Western blot examination for PrPSc/PrP27-30 as outlined above. The bioassay experiment was terminated at 350 days after infection.

\section{Acknowledgments}

The skillful technical assistance of Manuela Friedrich, Kristin Kampf, Marion Joncic, Ramona Famulla, Nadine Rupprecht, and Tatjana Pfander is gratefully acknowledged. This work was supported in part by grants from the German Bundesministerium für Bildung und Forschung, the German Bundesministerium für Gesundheit und soziale Sicherung, and the Volkswagen Foundation.

Received for publication January 15, 2004, and accepted in revised form April 2, 2004.

Address correspondence to: Michael Beekes, Robert Koch-Institut (P26 - Pathogenese und Diagnostik Transmissibler Spongiformer Enzephalopathien), Nordufer 20, 13353 Berlin, Germany. Phone: 4930-4547-2396; Fax: 49-30-4547-2267; E-mail: BeekesM@rki.de.

Achim Thomzig and Walter Schulz-Schaeffer contributed equally to this work.
7. Pattison, I.H., and Millson, G.C. 1962. Distribution of the scrapie agent in the tissues of experimentally inoculated goats. J. Comp. Pathol. 72:233-244.

8. Brown, P., et al. 1994. Human spongiform encephalopathy: the National Institutes of Health series of 300 cases of experimentally transmitted disease. Ann. Neurol. 35:513-529.

9. Bradley, R. 2001. Bovine spongiform encephalopathy and its relationship to the new variant form of Creutzfeldt-Jakob disease. In Prions: A challenge for science, medicine and public health system. H.F. Rabenau, J. Cinatl, and H.W. Doerr, editors. Karger. Basel, Switzerland. 105-144.

10. European Commission. 2002. Update of the opinion on TSE infectivity distribution in ruminant tissues.Outcome of discussions. http://www.europa. eu.int $/ \mathrm{comm} / \mathrm{food} / \mathrm{fs} / \mathrm{sc} / \mathrm{ssc} /$ outcome_en.html.

11. Bosque, P.J., et al. 2002. Prions in skeletal muscle. Proc. Natl. Acad. Sci. U. S. A. 99:3812-3817.

12. Thomzig, A., Kratzel, C., Lenz, G., Kruger, D., and
Beekes, M. 2003. Widespread PrPSc accumulation in muscles of hamsters orally infected with scrapie. EMBO Rep. 4:530-533.

13. Bartz, J.C., Kincaid, A.E., and Bessen, R.A. 2003. Rapid prion neuroinvasion following tongue infection. J. Virol. 77:583-591.

14. Glatzel, M., Abela, E., Maissen, M., and Aguzzi, A. 2003. Extraneural pathologic prion protein in sporadic Creutzfeldt-Jakob disease. N. Engl. J. Med. 349:1812-1820.

15. Kovacs, G.G., et al. 2004. Creutzfeldt-Jakob disease and inclusion body myositis: abundant diseaseassociated prion protein in muscle. Ann. Neurol. 55:121-125.

16. Kimberlin, R.H., and Walker, C.A. 1979. Pathogenesis of mouse scrapie: dynamics of agent replication in spleen, spinal cord and brain after infection by different routes. J. Comp. Pathol. 89:551-562.

17. Kimberlin, R.H., and Walker, C.A. 1980. Pathogenesis 
of mouse scrapie: evidence for neural spread of infection to the CNS. J. Gen. Virol. 51:183-187.

18. Kimberlin, R.H., and Walker, C.A. 1982. Pathogenesis of mouse scrapie: patterns of agent replication in different parts of the CNS following intraperitoneal infection. J. R. Soc. Med. 75:618-624.

19. Kimberlin, R.H., and Walker, C.A. 1989. Pathogenesis of scrapie in mice after intragastric infection. Virus Res. 12:213-220.

20. Hadlow, W.J., Kennedy, R.C., and Race, R.E. 1982. Natural infection of Suffolk sheep with scrapie virus. J. Infect. Dis. 146:657-664.

21. Beekes, M., McBride, P.A., and Baldauf, E. 1998 Cerebral targeting indicates vagal spread of infection in hamsters fed with scrapie. J. Gen. Virol. 79:601-607.

22. McBride, P.A., and Beekes, M. 1999. Pathological $\mathrm{PrP}$ is abundant in sympathetic and sensory ganglia of hamsters fed with scrapie. Neurosci. Lett. 265:135-138

23. Beekes, M., and McBride, P.A. 2000. Early accumulation of pathological PrP in the enteric nervous system and gut-associated lymphoid tissue of hamsters orally infected with scrapie. Neurosci. Lett. 278:181-184

24. McBride, P.A., et al. 2001. Early spread of scrapie from the gastrointestinal tract to the central nervous system involves autonomic fibers of the splanchnic and vagus nerves. J. Virol. 75:9320-9327.

25. van Keulen, L.J., Schreuder, B.E., Vromans, M.E., Langeveld, J.P., and Smits, M.A. 2000. Pathogenesis of natural scrapie in sheep. Arch. Virol. Suppl. 16:57-71. 26. Schulz-Schaeffer, W.J., Fatzer, R., Vandevelde, M., and Kretzschmar, H.A. 2000. Detection of $\operatorname{PrP}(\mathrm{Sc})$ in subclinical BSE with the paraffin-embedded tissue (PET) blot. Arch. Virol. Suppl. 16:173-180.

27. Bons, N., et al. 1999. Natural and experimental oral infection of nonhuman primates by bovine spongiform encephalopathy agents. Proc. Natl. Acad. Sci. U. S. A. 96:4046-4051.

28. Williams, E.S., and Miller, M.W. 2000. Pathogenesis of chronic wasting disease in orally exposed mule deer (Odocoileus hemionus): preliminary results [abstract]. In Proceedings of the 49th Wildlife Disease Association Conference. June 4-8. Jackson, Wyoming, USA. 29.

29. Sigurdson, C.J., Spraker, T.R., Miller, M.W., Oesch, B., and Hoover, E.A. 2001. PrP(CWD) in the myenteric plexus, vagosympathetic trunk and endocrine glands of deer with chronic wasting disease. J. Gen. Virol. 82:2327-2334.

30. Haik, S., et al. 2003. The sympathetic nervous system is involved in variant Creutzfeldt-Jakob disease. Nat. Med. 9:1121-1123.

31. Liddell, E.G.T., and Sherrington, C.S. 1925. Recruitment and some other factors of reflex inhibition. Proceedings of the Royal Society. Series B. 97:488-518.

32. Hirokawa, N., and Heuser, J.E. 1982. Internal and external differentiations of the postsynaptic membrane at the neuromuscular junctions. J. Neurocytol. 11:487-510.

33. Rohwer, R.G. 1984. Virus like sensitivity of the scrapie agent to heat inactivation. Science. 223:600-602.
34. Moynagh, J., and Schimmel, H. 1999. Tests for BSE evaluated. Nature. 400:105.

35. 1999. WHO infection control guidelines for transmissible spongiform encephalopathies. 1999. Report on a WHO consultation. Geneva, Switzerland.

36. Beekes, M., Mielke, M., Pauli, G., Baier, M., and Kurth, R. 2004. Aspects of risk management of nosocomial transmission of classical and variant Creutzfeldt-Jacob disease with special attention to German regulations. In Prions. A challenge for science, medicine and public health system. H.F. Rabenau, J. Cinatl, and H.W. Doerr, editors. Volume 11. 2nd revised edition. Karger. Basel, Switzerland. 117-135.

37. Beekes, M., et al. 1995. Western blot mapping of disease-specific amyloid in various animal species and humans with transmissible spongiform encephalopathies using a high-yield purification method. J. Gen. Virol. 76:2567-2576.

38. Kascsak, R.J., et al. 1987. Mouse polyclonal and monoclonal antibody to scrapie-associated fibril proteins. J. Virol. 61:3688-3693.

39. Schulz-Schaeffer, W.J., et al. 2000. The paraffinembedded tissue blot detects $\operatorname{Pr} \mathrm{P}(\mathrm{Sc})$ early in the incubation time in prion diseases. Am. J. Pathol. 156:51-56.

40. Kimberlin, R.H., and Walker, C.A. 1977. Characteristics of a short incubation model of scrapie in the golden hamster. J. Gen. Virol. 34:295-304.

41. Prusiner, S.B., et al. 1982. Measurement of the scrapie agent using an incubation time interval assay. Ann. Neurol. 11:353-358. 